\title{
Preliminary Study on SED Distribution of Tactile Sensation in Fingertip
}

\author{
Huiling Chen ${ }^{1}$, Liguo Shuai ${ }^{1}$, Weihang Zhu ${ }^{2}$ \\ ${ }^{1}$ School of Mechanical Engineering, Southeast University, Nanjing 211189, China \\ ${ }^{2}$ Department of Industrial Engineering, Lamar University, Beaumont, TX77710, USA
}

\begin{abstract}
For investigating the effects of stimuli on the deformations within the soft tissues of fingertips and the dependence of the tactile sensation on the deformations under pressure, in this paper, a finite element model is developed, to simulate the procedure of a fingertip touching a sharp wedge. Characteristics of the strain energy density (SED) distribution within the soft issues are analyzed. Simulation results show that the soft tissues of fingertips are very sensitive to stimuli, and the spatial distribution characteristics of strain energy density within soft tissues can best explain the evoked charging rate of mechanoreceptors.
\end{abstract}

\section{Introduction}

Human being skin has the ability of sensing physical stimulation such as pressure, vibration, temperature etc. When the fingertip touches an object, the tactile receptors under the skin will respond to the deformation of the skin and transmit electrical impulses to the brain through the peripheral nerve system. The main features of the impulses, and the frequencies of the nerve impulses, are associated with the mechanical environment of the tactile receptors, e.g. stress and strain within the skin, the strain energy density around the receptors. The study on the biomechanics of the fingertip perception is significant to understand the mechanism of tactile sensation and sens ory threshold.

As to the discrimination of one or two-point tactile stimuli and pathology of function disorders within soft tissue in the fingertip, there are two classical models, the physical model [1-2] and the structural model. The structural model refers to a linear elastic half-space models [3-4] and multilayer finite element (FE) models. The physical model is based on the quasi-viscoelastic constitutive equation. Similar with the physical model, linear elastic half- space models also simulate the total deformation characteristics of the skin in fingertip under load. However, it always neglects the multilayer performance of the fingertips, in this case, the model predictions may not agree well with the experiments, so linear elastic half-space model is unsuitable to simulate the local deformation of the soft tissues around to mechanoreceptors. The finite element model based on anatomical structure of fingertip can overcome the main shortcomings in other models. With the finite element model, Wu [5] made a research on soft tissue and bone injury in the fingertip. Maeno [6] also analyzed the complexity and the fine structure of finger skin (such as epidermal ridges, papillate) under deformation within the soft tissue. Simulation results by FE models show the feasibility of FE models to simulate the response of soft tissues to loading. In most FE models, the skin and subcutaneous tissue layer is defined as the linearly elastic. Although the linearly elastic model can get similar results with experimental data and reach a high efficiency in the case of small deformation mechanical stimulation, the skin layers and the subcutaneous tissues are factually nonlinear and time-dependent [7-9] and the response to the mechanical load simulation of the fingertip should also be nonlinear and time-dependent, respectively. Thus, the simple linear model has some limitations.

In this paper, a nonlinear finite element model of a multi-layer fingertip structure is proposed. With this model, the SED distribution is analyzed under a linear load. This paper analyzed the interaction model of the finger-tip with a sharp wedge (linear load), and the sensitivity of the model to weak stimuli is also discussed.

\section{Fingertip Model}

Anatomical structure of the fingertip is a multilayer structure including the epidermis, dermis, subcutaneous tissue, bone and nail, with the distribution of different types of mechanoreceptors inside it. Markel's discs are located in the epidermis, Meissner corpuscles and Ruffini endings are in the dermis. Pacini corpuscles are in the dermis and subcutaneous tissue. In previous studies $[1,2,10]$, results showed that the epidermis presents hyper-elastic and dermal tissue is linearly viscoelastic when skin is of large indentation. Zhang J et al. [11] pointed out that the mechanical behavior of the skin and the subcutaneous tissue can be simulated using the nonlinear biphasic constitutive model [12]. On the bas is of this theory, Wu [13] built a two-dimensional FEM model for a finger. In the model, the skin tissue is 
assumed to be hyperelastic and viscoelastic, and the subcutaneous tissue is considered to be a nonlinear, biphasic material co mposed of a hyperelastic solid and an inviscid flu id phase, and the nail and bone are considered to be linearly elastic. The proposed model by $\mathrm{Wu}[13]$ has the advantages in the ability to predict the deflection profile of the fingertip surface, the stress and strain distributions within the soft tissue, and the dynamic response of the fingertip to mechanical stimuli. However, when solving complex problems, the model is prone to be non-convergence and low efficiency, which may be due to its complexity. As we know, when the skin suffers a small deformation, the epidermis can be considered linearly elastic because of its small thickness.

In reference to physiological anatomy of the fingertip, we established a two-dimensional finite element model of a cross-section of the index fingertip. The outline of the model is regarded as a plaster contour based on [6]. The size of the index finger is measured by a reading microscope and the geometry can be mapped to an ellipse with a long axis of $17.44 \mathrm{~mm}$ and a short axis of $13.06 \mathrm{~mm}$, as shown in Figure.1. The bone structure is also simplified as an ellipse, with the center coinciding with the outer contour in $\mathrm{X}$-axis direction and $2 \mathrm{~mm}$ far in $\mathrm{Y}$ axis direction. Each layer of the tissues is symmetrical with the bone in the width direction, and the tissue thickness between the bone and the pulp skin surface is assumed to be bigger than that between the bone and the nail. From outside to inside, the skin layers are as follows, epidermis with the thickness of $0.2 \mathrm{~mm}$; dermis with the thickness $1.0 \mathrm{~mm}$, subcutaneous tissue between the dermis and the bone, the nail with the thickness of $1.2 \mathrm{~mm}$. The whole model is symmetrical on the $\mathrm{Y}$ axis, and in order to improve computational efficiency, we adopt the half model for simulation. As the touch deformation is assumed not over $2.0 \mathrm{~mm}$ in the later analysis, the epidermis is treated as linearly elastic. In order to reach a result close to the practical mechanical property of the skin in fingertip, the dermis and the subcutaneous tissue are regarded as different hyperelastic and linearly viscoelastic. The properties of the material constitutive equations are quoted from literature [14], whose feasibility has been verified. The bone and the nail are considered to be linearly elastic with a large Young's modulus. The linearly elastic parameters of the epidermis, the bone and nail are based on literature [5-6], as in Table 1. The sharp wedge for the stimulation is simplified as an analytical rigid, with the widths of $0.05 \mathrm{~mm}$ and $1.0 \mathrm{~mm}$ on the tip and base, respectively, and a height of $2.0 \mathrm{~mm}$.

Table 1. Constants of linearly elastic tissues.

\begin{tabular}{|c|c|c|c|}
\hline & Epidermis & Bone & Nail \\
\hline Young's Modulus (MPa) & 0.136 & 17000 & 170 \\
\hline Passion Rate & 0.48 & 0.3 & 0.3 \\
\hline
\end{tabular}

Table 2(a). Material parameters characterizing hyperelasticity and viscoelasticity for the skin $(\mathrm{v}=0.40)$.

\begin{tabular}{|c|c|c|c|}
\hline & $\mathrm{i}=1$ & $\mathrm{i}=2$ & $\mathrm{i}=3$ \\
\hline$\alpha_{\mathrm{i}}$ & 1.3416 & 25.000 & -7.8571 \\
\hline$\mu_{\mathrm{i}}(\mathrm{MPa})$ & $6.3754 \times 10^{-3}$ & $1.8874 \times 10^{-4}$ & $-3.7709 \times 10^{-4}$ \\
\hline $\mathrm{g}_{\mathrm{i}}$ & 0.0864 & 0.2136 & - \\
\hline$\tau_{\mathrm{i}}(\mathrm{s})$ & 0.2136 & 8.854 & - \\
\hline
\end{tabular}

Table 2(a). Material parameters characterizing hyperelasticity and viscoelasticity for the skin $(\mathrm{v}=0.40)$.

\begin{tabular}{|c|c|c|c|}
\hline & $\mathrm{i}=1$ & $\mathrm{i}=2$ & $\mathrm{i}=3$ \\
\hline$\alpha_{\mathrm{i}}$ & -2.0623 & 25.000 & -3.4784 \\
\hline$\mu_{\mathrm{i}}(\mathrm{MPa})$ & $2.3583 \times 10^{-2}$ & $4.5408 \times 10^{-5}$ & $4.0878 \times 10^{-2}$ \\
\hline $\mathrm{g}_{\mathrm{i}}$ & 0.2566 & 0.2225 & - \\
\hline$\tau_{\mathrm{i}}(\mathrm{s})$ & 0.3834 & 4.6731 & - \\
\hline
\end{tabular}

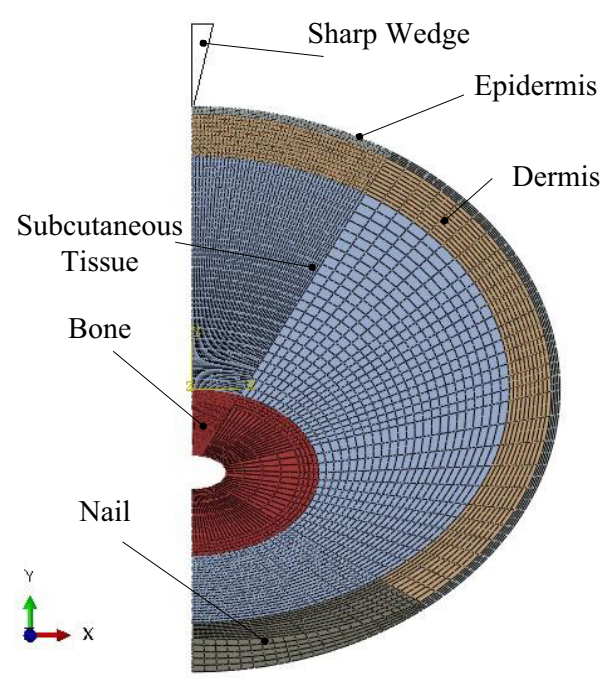

Figure 1. The contact model between the finger and the sharp wedge.

The fingertip was considered to be in contact with the sharp wedge. The biquadral, plain-strain elements were used in the FE modeling and the commercial finite element software package, ABAQUS/Standard (Version 6.11), was utilized for the analysis. The finite element model was shown in Figure. 1. The fingertip was fixed at the center on the nail surface, while the wedge was moved towards the skin surface in a ramp manner, such that a skin deformation of $1 \mathrm{~mm}$ was realized within a ramping period of $1 \mathrm{~s}$. The displacement of the wedge then remains constant. With this model, the nodes displacement, stress and strain distribution, strain energy density distribution and other field variables within the fingertip were calculated with the finite element method. 


\section{Simulation Results and Discussion}

The electrophysiological experiments suggest that the evoked discharge rate can best be explained by using of SED [16-19]. The spatial distribution characteristic of the SED within the tissues of fingertip is shown in Figure. 2(a) and (b). In the figures, A, B, C and D are the representatives of four different locations adjacent to the interfaces between epidermis and dermis, and that between dermis and subcutaneous tissue, respectively. Figure. 2(a) shows the distribution characteristics of SED along y-direction, from out-edge of the bone to the contact interface. It is seen that the SED has a sudden change from a layer to the next one, e.g, from A to B or fro $\mathrm{m} C$ to $\mathrm{D}$, and a gradual change within the same tissue layer, e.g, from $B$ to $C$, and then reaches a maximum at point $\mathrm{B}$ that locates at the interface between epidermis and dermis. From point $C$ to the out-edge of the bone, a maxima occurs at point $\mathrm{D}$ that locates at the interface between dermis and subcutaneous tissue. Because the load is very small, the SED mainly appears in the segment of AD line, while the mechanoreceptors are mainly concentrated in the AD segment and its vicinity as well. Figure. 2(b) shows the range of spatial response of the mechanoreceptors produced by the load. As it can be seen, the individual mechanoreceptor located in different positions has distinctive level of response, and the range of response is consistent to that of stimuli, i.e. contact outline. That's to say, the SED spatial distribution characteristics of the SED can help mechanoreceptors feel the stimuli outline feature and is related closely to the soft tissues' structural properties and stimuli features.

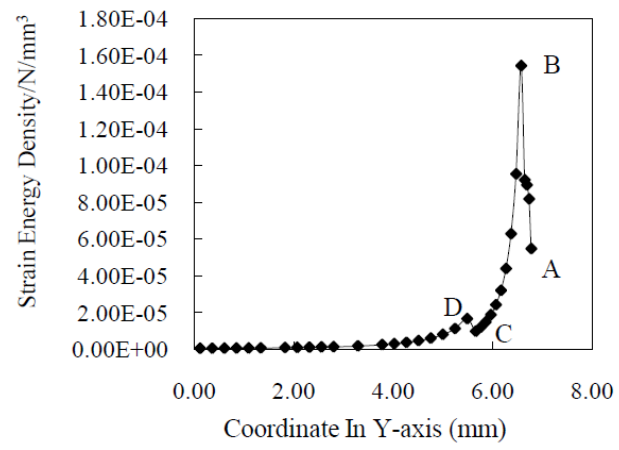

(a)

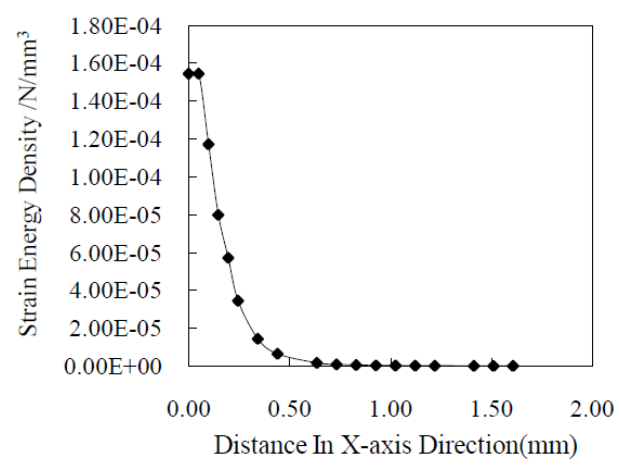

(b)

Figure 2. The distribution of SED within the tissues of fingertip.
The distributions of SED at depths of 0.20, 0.70, and $1.20 \mathrm{~mm}$ from the skin surface are shown in Figure. 3.

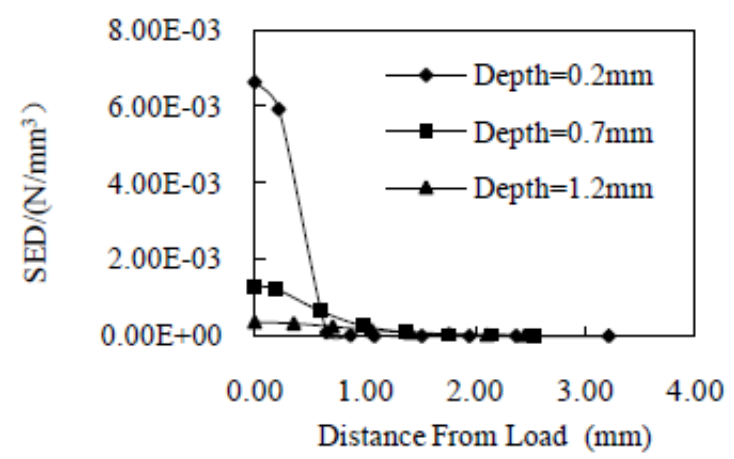

Figure 3. Predicted distributions of the SED in the soft tissue of the fingertip indented using a sharp wedge (at depths of $0.20,0.7$, and $1.20 \mathrm{~mm}$ within the tissue).

The value of SED is represented by the logarithmic strain. From Fig. 3 we can see that the larger the depth, the smaller the SED in value, within the $1 \mathrm{~mm}$. The SED are all approaching to 0 at the distance of over $1 \mathrm{~mm}$ from the stimulation location in the $\mathrm{X}$ direction.

\section{Conclusions}

The tactile discrimination of fingertip and distribution characteristics of contact strain is closely related to the performance of tactile sensation. However, because of the difficulty in implementing real-time measurement of the stress and strain within the fingertip, model simulation is a frequently used method to study the response to stimuli, based on the data from in-vitro experiments. This paper simulates the finger load response of the skin tissue in fingertip under the lineal pressure, the results show the tactile strain energy density spatial distribution can be well used to explain mechanoreceptors stimulated discharge characteristics; In addition, the result of the proposed finite element model under lineal load is consistent with the vitro experiments [6], and the strain energy density distribution of the epidermis and dermis around the mechanical receptors are mapped external shape of the wedge this model can be used to explore the mechanics of receptors under different types of mechanical stimuli.

\section{Acknowledgements}

This work is supported by the National Natural Science Foundation of China (61175069), the National Science Foundation's Course, Curriculum, and Laboratory Improvement (CCLI) program (0737173), and the Natural Science Foundation of Jiangsu Province of China (BK20131205).

\section{References}

1. D. Jindrich, Z. Yan, B. Theodore, et al. Non-linear viscoelastic models predict fingertip pulp force 
displacement characteristics during voluntary tapping. J Biomech, 2003, 36(4): 497-503.

2. J. Wu, R. Dong, W. Smutz, et al. Dynamic interaction between a fingerpad and a flat surface: experiments and analysis. Med Eng Phys, 2003, 25(3): 397-406.

3. J. Phillips and K. Johnson. Tactile spatial resolution III. a continuum mechanics model of skin predicting mechano receptor responses to bars, edges, gratings. J Neurophysiol, 1981, 46(6): 1204-1225.

4. M. Srinvasan, D. Dandekar. An investigation of the mechanics of tactile sense using two-dimensional models of the primate fingertip. J Biomech Eng, 1996, 118(1): 48-55.

5. J. Wu, R. Dong. Analys is of the contact interactions between fingertips and objects with different surface curvatures. Proc IMech E, 2005, 219(9): 89-103.

6. T. Maeno, K. Kobayashi and N. Yamazaki. Relationship between the structure of human finger tissue and the location of tactile receptors. JSME International Journal, 1998, 41(1): 94-100.

7. Y. Zheng and A. Mak. An ultrasound indentation system for biomechanical properties assessment of soft tissues in vivo. IEEE Bio med Eng, 1996;43:912918.

8. M. Rubin, S. Bodner and N. Binur. An elastic viscoplastic model for exc is ed facial tissues. ASME J Biomech Eng, 1998;120:686-9.

9. A.W. Wan . Biaxial tension test of human skin in vivo. Biomed Mater Eng ,1994;4:473-86.

10. Y. C. Fung. Biomechanics: Mechanical Properties of Living Tissues. Springer, New York, 1984.
11. J. Zhang, A. Mak, Huang L. A large deformation biomechanical model for pressure ulcers. ASME J Biomech Eng, 1997,119: 406-8.

12. V.C. Mow, S.C. Kuei and W.M. Lai, Armstrong CG. Biphasic creep and stress relaxation of articular cartilage: theory and experiment.ASME J Biomech Eng, 1980, 102:73-84.

13. J.Z. Wu, R.G. Dong, S. Rakheja, et al. A structural fingertip model for simulating of the biomechanics of tactile sensation. Medical Engineering \& Physics 26 (2004): 165-175.

14. J.Z. Wu and R.G. Dong, D.E. Welcome. Analysis of the point mechanical impedance of fingerpad in vibration. Medical Eng ineering \& Physics 28 (2006): 816-826.

15. M. Srinivasan. Surface deflection of primate fingertip under line load. J Biomech, 1989, 22:343-9.

16. K. Dandekar, B. Raju and M. Srinivasan. 3-d finiteelement models of human and monkey fingertips to investigate the mechanics of tactile sense. J Bio mech Eng, 2003, 125(5): 682-691.

17. K. O. Johnson. The role and functions of cutaneous mechanoreceptors. Curr Opin Neurobiol, 2001, 11(4): 455-461.

18. K. O. Johnson, T. Yoshioka and F. V. Bermudez. Tactile functions of mechano receptive afferents innervating the hand.J Clin Neurophysiol, 2000, 17(6): 539-558.

19. A. B. Vallbo and R. S. Johansson. Properties of cutaneous mechanoreceptors in the human hand related to touch sensation. Human Neurobiology, 1984, 3(1): 3-14. 